\title{
Environmental Tomography of Liquid Latex Suspensions in STEM
}

Juan Xiao, Lucian Roiban, Geneviève Foray and Karine Masenelli-Varlot

Univ Lyon, INSA-Lyon, CNRS UMR 5510, MATEIS, F-69621 Villeurbanne, France.

ESEM (Environmental Scanning Electron Microscopy) allows the observation of liquids under specific conditions of pressure and temperature. Moreover, when working in the transmission mode, i.e. in STEM (Scanning Transmission Electron Microscopy), nano-objects can be analyzed inside a liquid. Those acquired images are quite similar to those obtained in TEM (Transmission Electron Microscopy) using a closed cell [1]. By implementing a Peltier stage in the tomography device which was developed for the characterization of the 3D structure of non-conductive and low-contrast materials [2], the acquisition of image series of wet samples (wet-STEM tomography) [3]. Moreover, in situ evaporation of water can be performed to study the materials evolution from the wet to the dry state.

In our experiment, SBA-PMMA, a copolymer derived from styrene and metacrylic acid esters in aqueous solution, is chosen as the sample. The SBA particles has already included 3\% PMMA shell playing the role of steric surfactant. Some other surfactants can also be introduced to further stabilize the latex for some special applications [4].

In this work, we show series of tilted images that are acquired during in situ hydration / dehydration experiments with our improved device. Reconstructed volumes are obtained using the image processing software Etomo and ImageJ (see Figure 1), and the resolution is calculated using Fourier Shell Correlation (FSC). The influence of irradiation damage on the reconstructed volumes are discussed.

Afterwards, the volumes are segmented (see Figure 2) and quantified to follow the evolution of the microstructural parameters during water evaporation. This shows the potentialities of wet-STEM tomography for the characterization of suspensions from the wet to the dry state [5].

\section{References:}

[1] N de Jonge and FM Ross, Nature Nanotechnology 6 (2011), pp. 695-704.

[2] P Jornsanoh, Ultramicroscopy 111 (2011), pp. 1247-1254.

[3] K Masenelli-Varlot, A Malchère, Microscopy and Microanalysis 20 (2014), pp. 366-375.

[4] J Faucheu et al, Langmuir 25 (2009), pp. 10251-10258.

[5] The authors acknowledge the Consortium Lyon-Saint Etienne de Microscopie (CLYM) for the access to the microscope, the China Scholarship Council (CSC) and Institut Universitaire de France for financial support. 

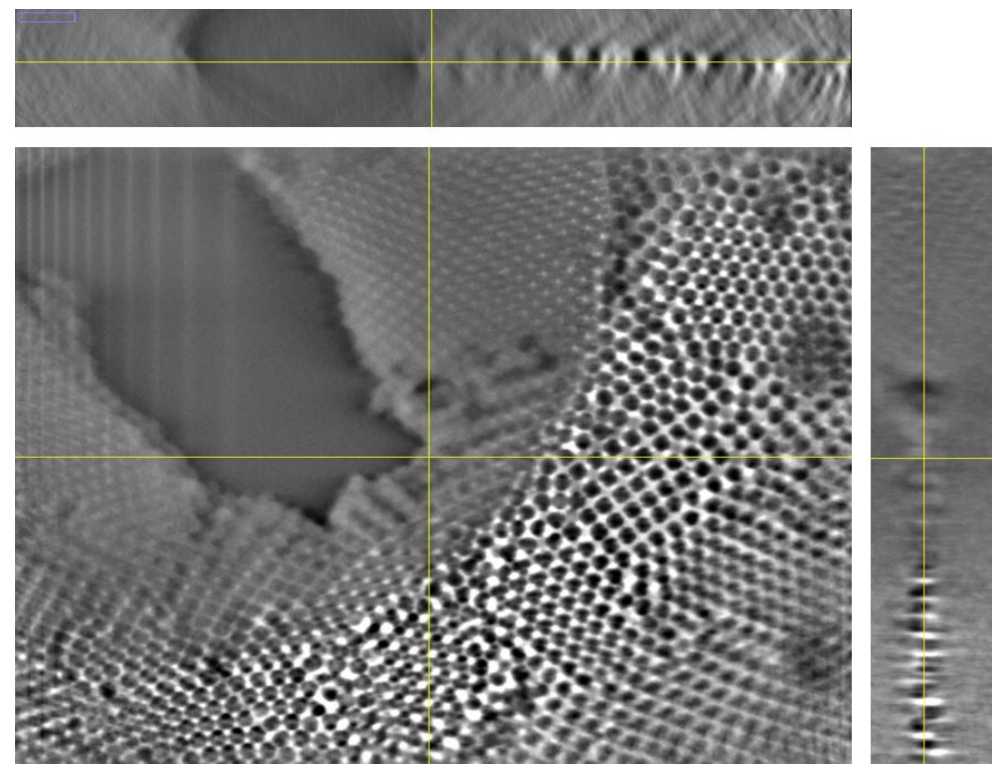

Figure 1. XY slice obtained on an area containing different layers of latex particles.

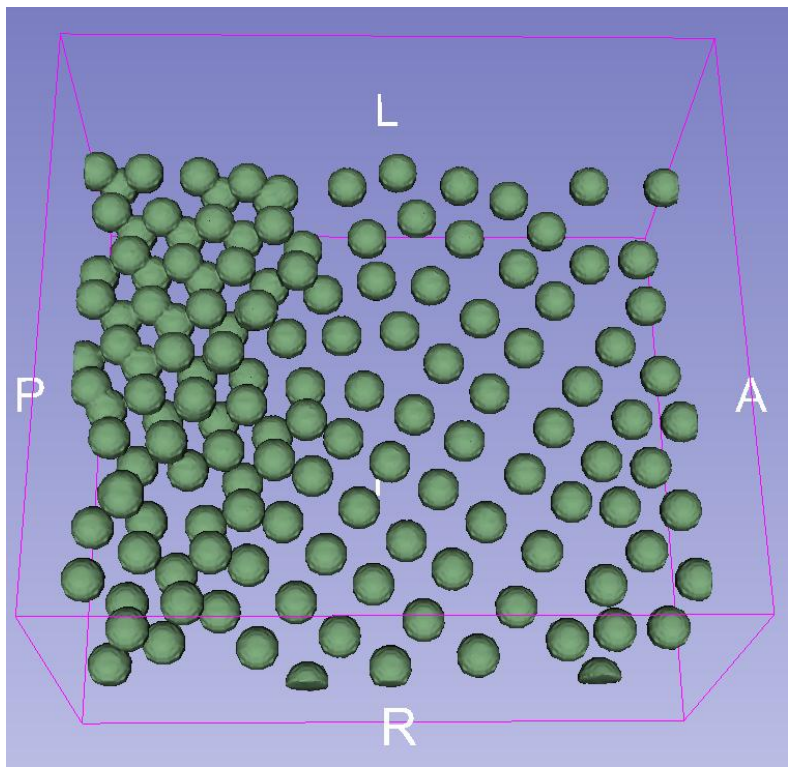

Figure 2. 3D arrangement of the latex particles obtained by segmentation of the reconstructed volume. 